\title{
A New Machine For Acquire Pavement Texture
}

\author{
João L. Vilaça*, Jaime C. Fonseca*, António M. Pinho**, Elisabete Freitas*** \\ * Industrial Electronic Department, **Mechanical Engineering Department, ***Department of Civil Engineering, University of \\ Minho, Campus Azurem 4800-058 Guimarães Portugal
}

\begin{abstract}
This paper presents a prototype machine for the acquisition and characterization of the macrotexture and megatexture of road surfaces. The development stages of the prototype machine involved: 3D data acquisition system configuration and calibration - based on laser triangulation technique, 3D surface reconstruction of the road surface and texture characterization using appropriated indicators, such as the Mean Profile Depth (MPD) and the Texture Profile Level (L), by applying different and complementary mathematical techniques. The prototype machine created is able to work in laboratory and in field, allowing an acquisition accuracy of 0.5 $\mathrm{mm}$. The contribution of this research is in developing a prototype machine capable of acquiring an extensive area road surface with high precision 3D data.
\end{abstract}

\section{INTRODUCTION}

Loads from vehicles alter the functional and structural characteristics of road pavements. The primary consequences are directly related not only to the loss of resistance of the pavement, but also to the users' comfort and safety. Therefore, those alterations need to be permanently observed. On the one hand at a network level, through compatible acquisition techniques considering high speeds and, on the other hand, at the project level, through techniques that allow acquiring detailed information.

Nowadays, those techniques use one spot measurement of a certain property through laser probes. The image acquisition, which is often submitted to a subsequent manual treatment, does not provide appropriate information for the establishment of 3D models of the observed surface. Achieving accurate 3D models for full-scale areas, at least as large as the tire-surface contact area, in a short period of time constitutes a step forward on the use of 3D structural analysis techniques, such as those based on Finite Element Method (FEM) and on the description of surface irregularities and defects, such as texture and segregation. In this context, the use of inverse engineering techniques (laser triangulation with laser line, among others) is more advantageous in relation to the methods used hitherto.

The prototype machine for the acquisition of road surfaces and their texture characterization presented in this paper was developed based on the triangulation principle, reaching a resolution of $0.5 \mathrm{~mm}$. This needed the construction of a mechanical prototype to be used in laboratory and in field. The mechanical prototype allows the displacement of an arm which holds the measurement system over the road surface. The measurement system arm is constituted by two cameras and one laser line. The precision of the measurements is assured by the calibration procedure developed for the measurement system.

The proposed prototype also implied the development of a software which contains a set of algorithms that combine computer vision and computer graphics methods for: a) 3D surface reconstruction from organized data sets (acquired data); b) data set treatment; c) data set analysis; d) data set export; e) road texture characterization, which includes two algorithms to calculate the indicators: Estimated Texture Depth (ETD) and Texture Profile Level (L).

The contribution of this research is in developing a prototype machine capable of acquiring an extensive area road surface with high precision 3D data, making possible, through the acquired data, the road texture characterization using two algorithms that allow calculating the ETD and L.

Section 2 summarizes the state-of-the-art of the existing systems which have been developed in order to characterize the texture of road surfaces.

Section 3 describes the 3D road texture acquisition system. In this section the mechanical system, the acquisition system set up, the calibration procedure used are discussed as well as the $3 \mathrm{D}$ acquisition software are presented.

Section 4 summarizes the theoretical foundations and the developed algorithms for the pavement characterization.

Finally, a comparative study between the proposed prototype machine and a contact measurement machine $(\mathrm{CMM})$ is presented in section 5. Furthermore, the results of the texture characterization of two different types of road surfaces by using the prototype machine are presented.

Conclusions and suggestions that aim to improve the developed system are presented in section 6 .

\section{RELATED WORK}

The functional quality of a road surface is determined by its texture and it is directly related to safety and driving comfort together with its structural quality, which is influenced by dynamic loadings. Texture also affects environment quality if vehicle emissions, wear, and noise are considered.

The surface texture is mostly determined by the selection of the materials (especially aggregates), the mixture design and the finishing techniques. Furthermore, surface texture changes throughout time due to traffic loading and, for that reason, it must be continuously monitored. Thus, within a certain texture range, monitoring techniques should allow for 
collecting detailed information easily at the project level, and be compatible with traffic speeds, at the network level.

Several methods to measure texture within the ranges of macrotexture and megatexture which partially fulfill those requirements have been developed.

At the network level, Ref. [1] present a summary of the existent commercial equipments and propose a mobile imaging system that incorporates multi-modality sensors for road surface mapping and inspection applications.

At the project level, emphasis will be given to equipments developed for characterizing the texture of road surfaces, such as the Contact Measure Equipment (CMM), the Circular Texture Meter (CTM) and the RoboTex, which have the same objective that the proposed prototype machine. These equipment/acquisition techniques go towards to a $3 \mathrm{D}$ description of the surface or object.

The CMM consists of a contact sensor assembled in a mechanical coordinate measure system. This data acquisition system is very precise. However, it is rather slow, expensive and restricted to materials resistant to mechanical contact [2].

The CTM and the RoboTex are examples of equipments that use computer vision techniques. Besides the fact of rapid data acquisition, computer vision allows for the acquisition of dense and precise data sets at high band widths. The CTM is a stationary apparatus that evaluates texture profile by circular samples, presenting difficulties on texture direction interpretation [3]. The RoboTex is a robotic texture apparatus built around LMI-Selcom's line laser, acquiring texture profiling at 1 × $1 \mathrm{~mm}$ sample intervals.

Image analysis techniques based on X-ray computed tomography that convert unabsorbed $\mathrm{X}$-ray to $2 \mathrm{D}$ images and then to 3D images. Projected Moiré Interferometry is also used [4].

These techniques represent an important advance for the acquisition of essential information for characterizing the texture of road surfaces. Nevertheless, they are expensive and the software tools provided to characterize the pavement texture are insufficient. Thus, the development of the TexScan, prototype equipment devoted to the characterization of the texture of road surfaces, is intended to overcome some of the limitations presented above.

\section{D ROAD TEXTURE ACQUISITION}

The implementation of the prototype machine had comprised three main phases. In the first phase, the mechanical structure and the control system were worked out in order obtain a safe self-mobile equipment. In the second phase, a calibration method for the data acquisition system was implemented. In the last phase, was developed a $\mathrm{VC}++$ software which acquires, processes, analyzes and exports the road pavement data.

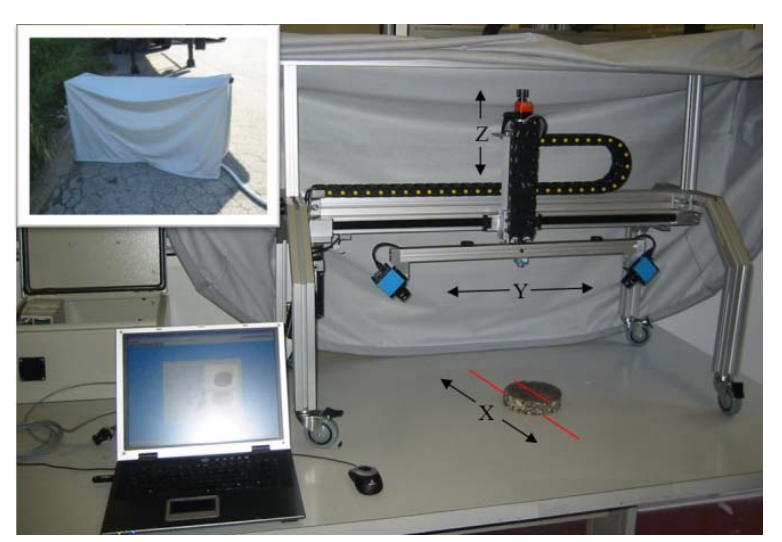

Figure 1. 3D road surface acquisition system composed of a mechanical prototype, a data acquisition system, a motion control system and a laptop.

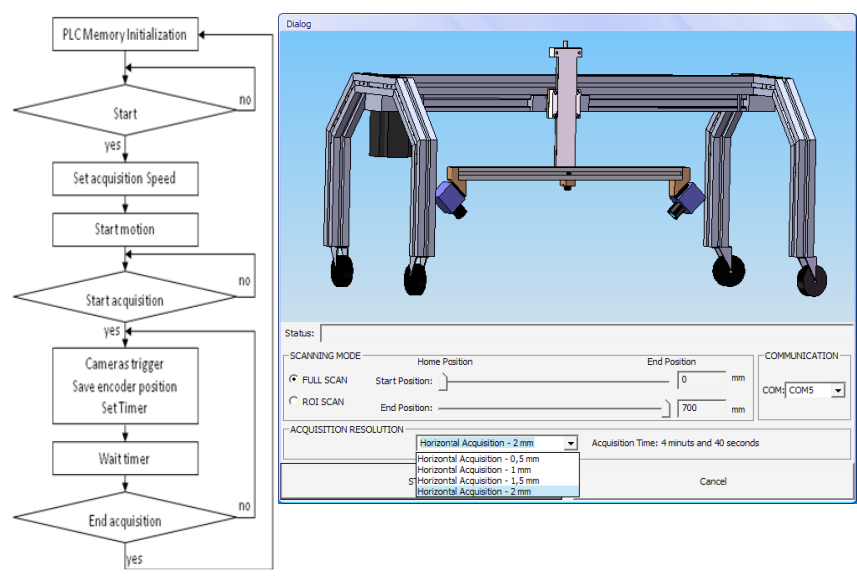

Figure 2. a) PLC pseudo algorithm; b) 3D texture acquisition software: acquisition setup dialog.

\section{TextScan Mechanical Prototype and Motion Control System}

The mechanical prototype is constituted by two mechanical axes which move in $\mathrm{Z}$ and $\mathrm{Y}$ directions (Fig. 1). The function of the $\mathrm{Z}$ axis is to set the height of the data acquisition arm (laser line and two cameras). This regulation is important to keep the road pavement surface inside the data acquisition system vision field, because laboratorial and field samples may have different heights. It is executed manually through the rotation claw coupled to the axis spindle. The function of the $\mathrm{Y}$ axis (belt type) is moving the $\mathrm{Z}$ axis along a certain length. The maximum $\mathrm{Y}$ course is $700 \mathrm{~mm}$, the axis is coupled to a step motor (Berger Lahr: VRDM368/50LWCOO), worked by a driver (Twin Line: TLD011F) with micro-step mode, in order to make the movement of the axis soft.

The motion of the prototype machine is controlled by a Programmable Logical Controller (PLC) (Omron: CPM1A10CDT-V-V1). The PLC controls the machine movements and ensures its physical limits, disabling the machine motion device every time that an end limit sensor is activated. Fig. 2 a) shows the algorithm implemented in the PLC. 
The motion speed (directly associated to the system acquisition resolution) and the sample lengths are setups supplied by the user (Fig. 2. b)). The user can select the full scanning mode or the ROI scanning mode. The first one acquires data over the total acquisition length $(700 \mathrm{~mm})$. The second one executes a partial acquisition. In this mode, the user defines the start and end points. Relatively to the acquisition resolution, the user can select one of four values: $0.5 \mathrm{~mm}, 1 \mathrm{~mm}, 1.5 \mathrm{~mm}$ and $2 \mathrm{~mm}$. The selection of the system resolution will directly affect the acquisition speed. These setups are sent from the PC to the PLC using the serial protocol RS232. To establish the communication with the PLC Omron the Host-link protocol was implemented using $\mathrm{C}++$ language.

After setting the acquisition speed, the PLC controls the trigger signal of the two cameras. The value of the axis encoder is saved in a memory area each time the trigger is activated.

The data acquisition system (laser and cameras) is supported by a mechanical structure built in aluminum profiles, with a base module structure assembled to free wheels, for an easier transportation. The machine was developed to be used in laboratory and on field. It has a transportable cover which ensures adequate acquisition conditions (Fig. 1 top-left corner).

\section{TextScan data acquisition system}

The computer vision area includes accurate measure methods where data acquisition is made through structured light and cameras. These methods are based on geometric relations (triangulation) that can be set between the acquisition devices (cameras) and illumination (laser) and the surfaces to scan. The use of line lasers in this type of systems allows for getting high-density data (that could be programmable) and a most flexible approach for complex surfaces.

This type of methods is the basis of the data acquisition system presented hereafter. The developed system is constituted by a laser of line (lens with opening of $120^{\circ}, 3 \mathrm{~mW}$ of power and a wavelength of $735 \mathrm{~nm}$ ) and two firewire cameras (The ImagingSource: DMK 31BF03) with a resolution of $1024 \times 768$ and with $8 \mathrm{~mm}$ lenses. The use of two cameras instead of one is due to the fact that road surfaces are complex and therefore may hide the laser line visualized by the camera (occlusion problem).

Mechanically the acquisition arm is settled as follows: the laser is equally distant from both cameras. The cameras make a $45^{\circ}$ angle with the plane defined by the laser line. More details of the acquisition arm are shown in Fig. 3 a).

The calibration of this type of systems is usually hard and time consuming task. In this specific work the calibration procedure used is the one in [5], which takes the laser plane as calibration plan, what simplifies its implementation. The calibration procedure is divided into three steps: image horizontal calibration, image vertical calibration and image

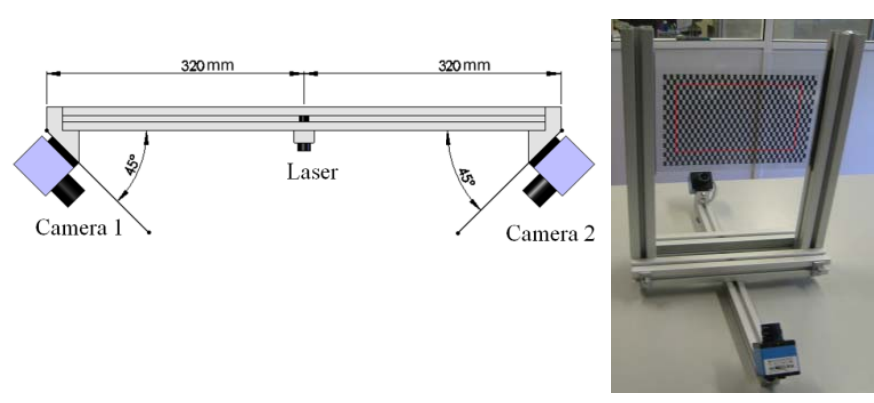

Figure 3. Data acquisition system: a) mechanical disposition of the acquisition arm; b) acquisition arm assembled to the calibration support.

coordinates correspondence with real-world coordinates. All steps are based on polynomial interpolation of collected pattern points (Fig. 3 b)). The first two steps execute the acquired images of both cameras in parallel, what results in two new images corrected horizontally and vertically. The third step is made after a logical OR between the two images that are horizontally and vertically corrected. The authors also propose an algorithm for automatic detection of laser lines based on the image vertical first derivative, which was also used.

Relatively to the system proposed in [6], the angle of both cameras was modified, of $60^{\circ}$ to $45^{\circ}$, to improve the visualization of deeper areas on the surface. With this modification an improvement of the acquired data was verified. Nevertheless, the system resolution decreased, what was overcome by increasing the camera resolution. The data acquisition system vision field was defined to 200 (axis X) x 200 (axis Z) $\mathrm{mm}$ with a resolution of $0.5 \times 0.5 \mathrm{~mm}$.

This software module was implemented in $\mathrm{VC}++$ and it was integrated in the machine acquisition software. The software is called whenever the trigger signal of the cameras is activated by the PLC. Concisely, the algorithm calibrates and extracts the laser line view by the cameras and associates the line points to the scanner arm position (saved in the same instant of the PLC trigger shot), read from a PLC memory position.

\section{TextScan software}

The software of the TexScan was implemented in $\mathrm{VC}++$. It is responsible for the following tasks: data acquisition, data processing, data analysis and data export for the formats .txt and .stl (STereo Lithography).

Data are acquired line by line. Each line is constituted by a set of points and represents $2 \mathrm{D}$ height map ( $\mathrm{z}$ and $\mathrm{x}$ coordinates) of the acquired sample. The result of the acquisition process is a $3 \mathrm{D}$ height map, where the $\mathrm{y}$ coordinate of each line is given by the axis encoder value.

Once the acquired data are organized in space, the developed software uses an algorithm (Fig. 4) to produce a triangular mesh. This algorithm uses the max-min angle 


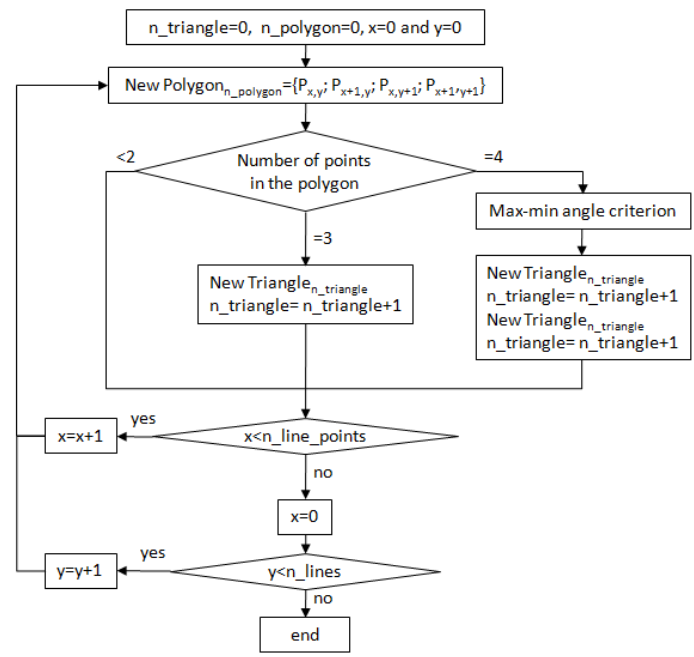

Figure 4. Algorithm of the 3D surface reconstruction from an organized data set, where: $n \_$triangle - number of triangles; $n \_$polygon - number of polygons; $\mathrm{P}$ - point; Polygon - polygon constituted up to four points; Triangle - triangle constituted by three points; $n \_$line_points - number of points in each line; $\mathrm{n} \_$lines - number of scanner lines.

\begin{tabular}{|l|l|l|}
\hline 0 & 0.2 & 0 \\
\hline 0.2 & -1.8 & 0.2 \\
\hline 0 & 0.2 & 0 \\
\hline 1
\end{tabular} \begin{tabular}{|l|l|l|}
\hline 1 & 2 & 1 \\
\hline 2 & 4 & 2 \\
\hline 1 & 2 & 1 \\
\hline
\end{tabular}

\begin{tabular}{|l|l|l|l|l|}
\hline 2 & 7 & 12 & 7 & 2 \\
\hline 7 & 31 & 52 & 31 & 7 \\
\hline 12 & 52 & 127 & 52 & 12 \\
\hline 7 & 31 & 52 & 31 & 7 \\
\hline 2 & 7 & 12 & 7 & 2 \\
\cline { 1 - 6 }
\end{tabular} \begin{tabular}{|l|l|l|}
1 & 1 & 1 \\
\hline 1 & 1 & 1 \\
\hline
\end{tabular}

Figure 5. Smoothing masks: a) Laplacian 3x3, b) Gaussian 3X3, c) Gaussian $5 \times 5$ and d) Average $3 \times 3$.

criterion [6] for setting triangles from a quadrangular polygon.

The software provides three types of mesh smoothing filters for data processing. Mesh smoothing is a technique that adjusts the point-coordinates of a data set. The objective of the mesh smoothing is the improvement of the appearance of a mesh and/or the improvement of the shape of the data set. The most used smoothing technique is the basic Laplacian smoothing, which is based on the Laplacian flow. The discrete implementation is achieved by multiplying the Laplacian operator mask with a $3 \times 3$ mask (Fig. 5 a)) to the data set. Based on this technique a set of smoothing surface filters were developed. A Gaussian filter with $3 \times 3$ (Fig. 5 b)) and $5 \times 5$ (Fig. 5 c)) neighboring and a simple $3 \times 3$ average filter (Fig. 5 d)).

To preserve the data set topology, a normal vector analysis is executed in each neighborhood of the analyzed point. If the point in analysis presents neighborhood normal vectors with different orientations (this difference should be higher than $30^{\circ}$ ) the smoothing technique is not applied to the point. The filter which showed the best results was the Gaussian $5 \times 5$.

Furthermore, this software provides the user with the possibility of selecting a region of interest, (circular or rectangular) of the acquired sample for data analysis (maximal, minimal and height values) and data export. In relation to the data export, the software disposes of two export formats: STL neutral file and TXT file. The STL file can be used to model the road by finite element analysis software. The TXT file is used to characterize the road texture in the software developed in Matlab, which is presented in the next section. The .txt file is saved with all the point lines of the acquired sample belonging to the select region of interest.

\section{D ROAD TEXTURE CHARACTERIZATION}

The profile of a road surface may be described by two coordinates: the distance along a certain travel direction, the amplitude which is normal to the surface plane [7] and the texture wavelength defined as the (minimum) distance between periodically repeated parts of the curve.

After the acquisition of the set of profiles, which constitute a 3D sample of the pavement, it is necessary to characterize them with appropriated indicators, such as the Mean Profile Depth (MPD) and the Texture Profile Level (L), using different and complementary mathematical techniques. They were implemented in Matlab version 7.1 using .m files. The whole Matlab processing is made by lines which are composed by the points obtained after the acquisition and processing effectuated by the acquisition software of the prototype machine.

\section{Mean Profile Depth and Estimated Texture Depth}

The depth of a profile is the difference in height between the profile and a horizontal line through the top of the highest peak (the peak level) within a distance along the surface of the same order of length as a typical tire-pavement interface. The MPD is calculated at a certain profile distance (base line), usually $10 \mathrm{~cm}$, as indicated in Fig. 6 and by (1) [7].

$$
M P D=\frac{\text { Peaklevel }\left(1^{\text {st }}\right)+\operatorname{Peaklevel}\left(2^{\text {nd }}\right)}{2}-\text { Averagelevel }
$$

For comparison with volumetric techniques, such as the "Sand Patch Test", the MPD may be transformed through a conversion (2) [8] into an ETD.

$$
E T D=0.2+0.8 * M P D
$$

With this system the scanning is made by lines. Therefore, the MPD of the sample is determined by averaging the mean profile depth calculated for each line through (1). The ETD is calculated then through (2).

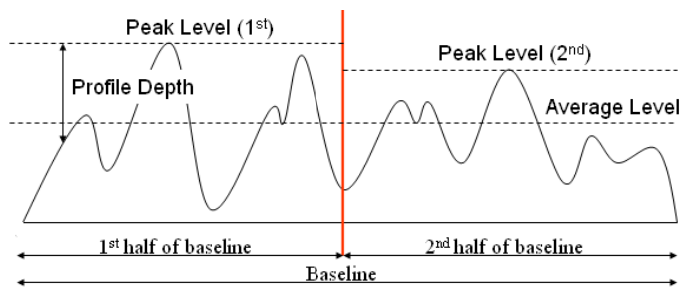

Fig. 6. Illustration of the base line concept, peak level, profile depth and average level. 


\section{EXPERIMENTAL RESULTS}

To demonstrate the capabilities of the proposed prototype machine, two types of analysis were effectuated. The first one is intended to certify the acquisition system of the prototype machine. For this analysis, a sample of road pavement was used for comparing the results obtained by the prototype machine acquisition system and by a high precision CMM (Mitutoyo, Class 0, Precision $0.1 \mu \mathrm{m}$ ). The second analysis presents the characterization of two types of road surface textures, through the determination of the Mean Profile Depth (MPD) and of the Texture Profile Level (L).

\section{Data acquisition system error results}

The possibility of a practical application of the developed acquisition system in the dimensional inspection of road surface, regarding macrotexture and megatexture, is demonstrated by means of a comparative study of a profile line carried out with a CMM machine and the acquisition system (Fig. 7). The horizontal sampling was carried out every of $1 \mathrm{~mm}$. The sample collected has a complex profile which constitutes a drawback to the profile acquisition in some narrow concavities captured by the CMM machine, as shown in the intervals: $\{23,28\}$ and $\{30,40\}$. Tables 1 and 2 show the statistical comparison between the two acquisition systems. It is possible to draw the following conclusions:

- The mean value of the CMM system $(0.36 \mathrm{~mm})$ is higher than the mean value of the developed system. This fact may be influenced by two factors: an error by the acquisition system and CMM difficulty in reaching narrow concavities.

- The maximal vertical value of acquisition points has almost the same value for both systems. The same happens for the minimal vertical value.

- The maximal acquisition point has almost the same value for both systems.

- The standard deviation is bigger in the developed prototype machine than in the CMM system; this fact could be influenced for the same factors that influenced the mean value.

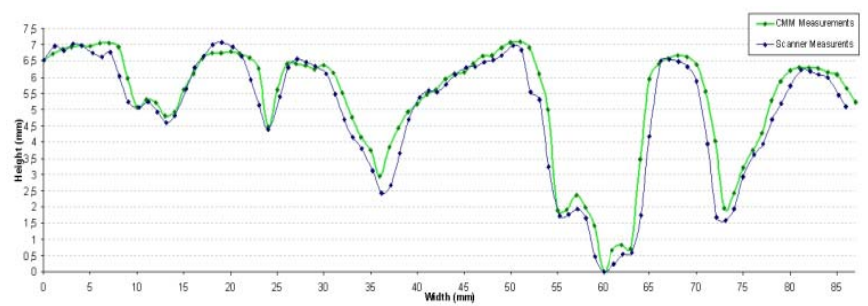

Figure 7. This graph establishes a comparison between the lines whose points were acquired by the acquisition system used and the CMM over road profile surface.
TABLE I

COMPARISON BETWEEN POINTS ACQUIRED BY CMM AND THE ACQUISITION SYSTEM USED

\begin{tabular}{|c|c|c|c|c|}
\hline $\begin{array}{c}\text { Measurement } \\
\text { System }\end{array}$ & Mean & Min & Max & STD \\
\hline $\begin{array}{c}\text { CMM } \\
\text { (unit:mm) }\end{array}$ & 5.29 & 0.00 & 7.09 & 1.76 \\
\hline $\begin{array}{c}\text { Developed } \\
\text { system } \\
\text { (unit:mm) }\end{array}$ & 4.93 & 0.01 & 7.08 & 1.92 \\
\hline
\end{tabular}

TABLE 2

ERRORS IN DISTANCES BETWEEN POINTS ACQUIRED BY CMM AND THE DEVELOPED SYSTEM

\begin{tabular}{|c|c|c|c|c|}
\hline Measurement & Mean & Min & Max & STD \\
\hline Error(unit:mm) & -0.13 & -0.24 & 0.05 & 0.07 \\
\hline
\end{tabular}

\section{Road texture characterization of two types of pavement}

The goal of the second analysis is the characterization of the macrotexture of four road samples using the developed prototype machine and the comparison of the obtained ETD values with the values calculated by the "Sand Patch Method". Samples A and B are made of "Porous Asphalt", which provides a rough texture, and samples $\mathrm{C}$ and $\mathrm{D}$ are made of "Dense Asphalt" which provides a smooth texture.

The "Sand Patch Method" is used to calculate the Mean Texture Depth (MTD) [10]. It uses a volumetric approach to measure the pavement macro texture. In this method, a known volume of glass beads is evenly spread over the pavement surface to form a circle, thus filling the surface with glass beads. The diameter of the circle is measured on four axes and the value averaged, determining the MTD, which is comparable to the ETD.

The four samples were acquired by the developed prototype machine using a precision of $0.5 \times 0.5 \times 0.5 \mathrm{~mm}$. After acquiring the samples, a circular region of interest (ROI) of $120 \mathrm{~mm}$ (diameter) was created in each sample. The diameter and centre of the selected ROI was the same that was used in the "Sand Patch Method". The points belonging to the selected ROI were then exported to be used in Matlab.
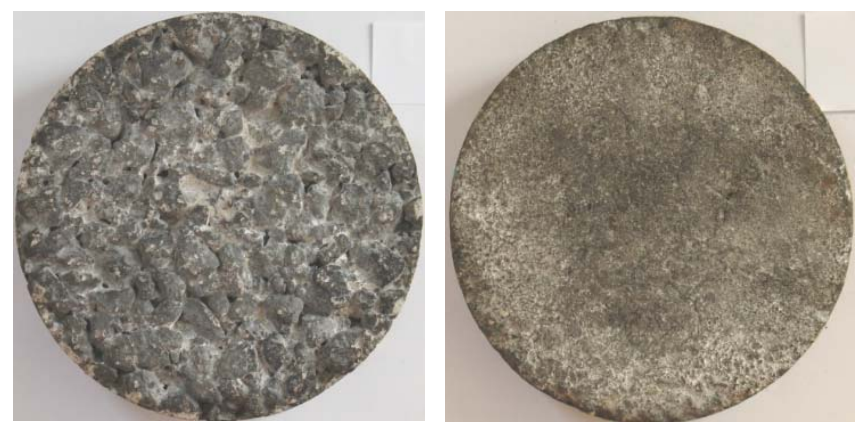

Figure 8. a) Sample A picture; b) Sample D picture. 


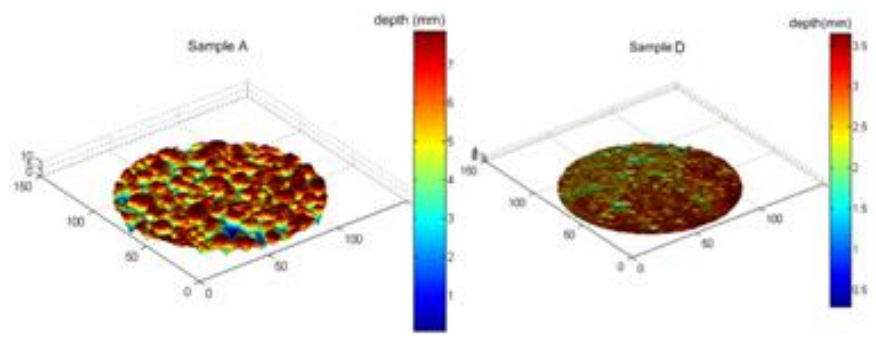

Figure 9. a) 3D surface visualization of the acquired sample A; b) 3D surface visualization of the acquired sample D

Figure 8 presents the aspect of the surface of the porous asphalt (Fig. 8 a)) and of the dense asphalt (Fig. 8 b)), the 3D acquired surface (represented by a color height map) (Fig. 9 a) and 9 b)). A sample of "Porous Asphalt" is represented in the left column and a sample of "Dense Asphalt" is represented in the right column. By analyzing the texture profile level chart of both samples, it is possible to verify that the frequency values of each sample are the same. Nevertheless, for each frequency, the texture level of the "Porous Asphalt" sample is higher than the texture level of the "Dense Asphalt" sample. This shows that it will be possible the use of the developed algorithm for the texture characterization by visualizing the surface of the road easily and by analyzing which frequencies suffer significant changes. The MTD and ETD values of the four samples calculated by the developed prototype machine and by the "Sand Patch Method" are presented in Table 3. The results show that the ETD values calculated by the TextScan are similar to the ones calculated by the "Sand Patch Method". Likewise, the relation between the ETD values calculated by the developed prototype machine and the MTD values calculated by the "Sand Patch Method" of both samples is practically the same (table 4), varying more in samples $C$ and D. This fact may be explained by the difficulty of application of the "Sand Patch Method" in this type of samples. The results obtained validate the use of the developed algorithm for this indicator in texture characterization.

TABLE 3

MPD AND ETD RESULTS

\begin{tabular}{|c|c|c|c|c|}
\hline \multicolumn{2}{|c|}{} & \multicolumn{2}{|c|}{ TexScan } & $\begin{array}{c}\text { "Sand Patch } \\
\text { Method" }\end{array}$ \\
\hline $\begin{array}{c}\text { Sample } \\
\text { name }\end{array}$ & Sample & MPD & ETD & ETD \\
\hline \multirow{2}{*}{$\begin{array}{c}\text { Porous } \\
\text { Asphalt }\end{array}$} & A & 1.0059 & 1.0047 & 0.9738 \\
\cline { 2 - 5 } Dense & B & 1.5162 & 1.4130 & 1.4126 \\
\cline { 2 - 5 } Asphalt & D & 0.2629 & 0.4130 & 0.4512 \\
\hline
\end{tabular}

TABLE 4

ETD RELATION BETWEEN PROTOTYPE EQUIPMENT AND "SAND PATCH METHOD"

\begin{tabular}{|c|c|c|c|c|c|}
\hline \multicolumn{2}{|l|}{} & $\begin{array}{c}\text { Sample } \\
\text { A }\end{array}$ & $\begin{array}{c}\text { Sample } \\
\text { B }\end{array}$ & $\begin{array}{c}\text { Sample } \\
\text { C }\end{array}$ & $\begin{array}{c}\text { Sample } \\
\text { D }\end{array}$ \\
\hline $\begin{array}{c}\text { ETDprototype } \\
\text { equipment / } \\
\text { ETDsand } \\
\text { patch method }\end{array}$ & 1.0317 & 1.0002 & 0.9153 & 0.9542 \\
\hline
\end{tabular}

\section{CONCLUSIONS AND FUTURE WORKS}

This paper presented different construction stages of a prototype machine to obtain, in $3 \mathrm{D}$, the irregularities of a road pavement surface and its texture characterization. A new mechanical configuration of the acquisition arm was adapted, and arm components with different characteristics, comparatively to the system presented by Vilaça et al, were used in a way to improve the acquisition of difficult visualization areas, that is present in this type of surfaces.

In the pavement characterization module, two algorithms were developed: one for the calculation of the ETD and another one for the determination of the texture profile level. Both algorithms were tested over two different types of road surfaces. The results validated the use of these algorithms to characterize the texture using the ETD and texture profile level indicators. The machine also comprises a simple and friendly user interface, as a result of the interconnection among the machine modules and the low acquisition times. A road sample of $700 \mathrm{~mm}$ length for $200 \mathrm{~mm}$ width, with a resolution of $0.5 \times 0.5 \mathrm{~mm}$, was acquired and post-processed in 280 seconds. Further developments from this work will include providing the machine with a module for the characterization of cracks that may develop on the road surface. Thus, the equipment will be able to determine the crack length, crack orientation, crack depth and maximum and medium crack opening.

\section{REFERENCES}

[1] Si-Jie Yu, Sreenivas R. Sukumar, Andreas F. Koschan, David L. Page and Mongi A. Abidi, 3D reconstruction of road surfaces using an integrated multi-sensory approach, Optics and Lasers in Engineering, Volume 45, Issue 7, July 2007, Pages 808-8.

[2] A. Woźniak, M. Dobosz, Metrological feasibilities of CMM touch trigger probes. Part I: 3D theoretical model of probe pretravel, Measurement, Volume 34, Issue 4, December 2003, Pages 273-286,

[3] Douglas I. Hanson, Brian D. Prowell, Evaluation of circular texture meter for measuring surface texture of pavements, NCAT Report 0405, September 2004.

[4] Ala Abbas, M. Emin Kutay, Haleh Azari, Robert Rasmussen, ThreeDimensional Surface Texture Characterization of Portland Cement Concrete Pavements, Computer-Aided Civil and Infrastructure Engineering 22 (3), 2007, 197-209,.

[5] João L. Vilaça, Jaime C. Fonseca, A. C. Marques Pinho, Stereo vision calibration procedure for 3D surface measurements, Annual Conference of the IEEE Industrial Electronics Society (IECON 2006) : proceedings, ISBN 1-4244-0136-4, 2006.

[6] B.K.Choi, H.Y.Shin, Y.I.Yoon and j.W.Lee, Triangulation of scattered data in 3D space, Computer-Aided Design, 20(5), 1988, pp. 239-248.

[7] ISO/CD 13473-5, Characterization of Pavement Texture by Use of Surface Profiles - Part 5: Measurement of megatexture, Draft standard ISO/CD 13473-5, International Organisation for Standardisation (ISO), Geneve, Switzerland.

[8] ISO 13473-1:1997, Characterization of Pavement Texture by Use of Surface Profiles - Part 1: Determination of Mean Profile Depth, International Organisation for Standardisation (ISO), Geneve, Switzerland. 